\title{
Dislocation Motion in Al-Mg Alloys in the Creep Region Characterized by Activation Volume
}

\author{
Hidenari Takagi \\ Division of Applied Physics, Department of General Education, College of Engineering, Nihon University, Koriyama 963-8642, Japan
}

\begin{abstract}
To characterize the thermal activation process of dislocation motion in the creep region, thermal activation parameters must be determined. However, the activation volume has never been used to directly understand dislocation motion in this region. In this study, the effective stress and activation volume during creep in Al-Mg solid-solution alloys were determined using indentation techniques. The obtained effective stress and activation volume corresponded to the results obtained from the uniaxial test. At the tested creep rate of $\dot{\varepsilon}_{1}=1 \times 10^{-5}-4 \times 10^{-3} \mathrm{~s}^{-1}$, the obtained activation volume decreased with increasing solute concentration, a trend opposite to that of the effective stress. Using this activation volume and previously reported computer simulation results of the interactions between dislocations and solute atoms, the thermal activation process of dislocation motion during creep in several $\mathrm{Al}-\mathrm{Mg}$ solid-solution alloys was investigated. The thermally activated dislocation length decreased with increasing stress and was smaller than that between the forest dislocations. The results indicated that dislocations during creep could overcome thermal obstacles when the corresponding effective stress was interpolated against the increase/decrease in thermally activated dislocation length, and each dislocation fragment on the length between forest dislocations moved individually through each thermal activation process. [doi:10.2320/matertrans.MT-M2020359]
\end{abstract}

(Received November 25, 2020; Accepted June 7, 2021; Published July 16, 2021)

Keywords: thermal activation process, dislocation, effective stress, indentation

\section{Introduction}

To understand the creep behavior and the rate-controlling mechanisms in metals and alloys, thermal activation parameters, such as the stress exponent, activation energy, thermal activation stress (effective stress), athermal activation stress (internal stress), and instantaneous plastic strain, are evaluated. ${ }^{1,2)}$ The stress exponent and activation energy are the main indices for understanding the rate-controlling mechanism of creep, e.g., dislocation motion, grain boundary sliding, and diffusion. ${ }^{1)}$ When the mechanism is dislocation motion, the obtained effective stress and instantaneous plastic strain identify whether the dislocation motion is high-speed or viscous. ${ }^{2)}$ However, the above parameters do not identify any internal activities regarding the dislocation motion, such as the dislocation length overcoming a thermal obstacle.

One of the ways to identify such activities is to evaluate the activation volume, which is related to the area swept by a dislocation of a certain length when it moves by a certain distance, as it attempts to overcome the thermal obstacle, driven by the effective stress and thermal energy. ${ }^{3,4)}$ This activation volume can be used to identify the type of thermal obstacle that is impeding dislocation motion in metals and alloys at low and intermediate temperatures. ${ }^{3)}$ There are many reported studies aimed at understanding dislocation motion and the interactions between dislocations and thermal obstacles based on the activation volume. ${ }^{3,4)}$ Recently, Choi et al. investigated the temperature-dependent mechanical behavior of $\mathrm{Cr}$ using obtained data, such as hardness, strain-rate sensitivity, and activation volume, and they discussed the role of dislocation in the plastic relaxation mechanisms. ${ }^{5)}$ Tanaka et al. assessed the temperature dependence of the yield stress, effective stress, and activation volume based on the $\mathrm{Cu}$ content of ultra-low carbon steel. $\left.{ }^{6}\right)$ They pointed out that an abrupt increase in the activation volume reflected a major difference in the thermally activated process. Moreover, the activation volume has been used to study the thermal activation process of dislocation motion to demonstrate the plastic deformation behavior of advanced materials such as ultrafine-grained materials ${ }^{7)}$ and highentropy alloys. ${ }^{8)}$ The abovementioned observations imply that the activation volume is essential for characterizing the thermal activation process of dislocation motion and for identifying the deformation mechanics of metals and alloys.

At high temperatures, at which creep occurs dominantly, activation volume is also evaluated.,.$^{3,4-11)}$ However, the activation volume is not used to directly understand the dislocation motion, such as identifying the length and distance of the thermal activation process. There is no simple theory for studying the thermal activation process due to the complexity of the contribution of thermal energy. ${ }^{4)}$ Recently, Curtin's group proposed a principle for predicting the solute strengthening of alloys using theoretical analyses and computer simulations, ${ }^{12-14)}$ which they performed based on the model of dislocation motion proposed by Labusch, ${ }^{15}$ ) assuming that moving dislocations interact simultaneously with several solute atoms. Additionally, they showed several theories regarding solute strengthening from low to high temperatures. Leyson and Curtin also elucidated the relationship between the thermally activated dislocation length and the moved distance at high temperatures. ${ }^{14)}$ Using the results reported by Curtin's group, dislocation motion during creep can be understood directly from an experimentally obtained activation volume. Therefore, the activation volume is essential for identifying creep deformation mechanisms, and this information can eventually help propose new ways of improving creep properties, such as creep strength and resistance.

In this study, we treated $\mathrm{Al}-\mathrm{Mg}$ solid solution alloys as the model material. It is known that the viscous glide of dislocations can be accomplished by an intake of thermal energy and effective stress. ${ }^{2,16,17)}$ This means that thermal obstacles exist, and dislocations must overcome these obstacles by the thermal activation process. Therefore, the 
evaluated activation volume should be related to the dislocation motion in the alloys. Here, the effective stress and activation volume during creep in $\mathrm{Al}-\mathrm{Mg}$ solid-solution alloys were evaluated based on information acquired using indentation techniques. To validate the obtained results, the results were compared with those obtained by the uniaxial testing method. In addition, the dependences of the effective stress and activation volume on the solute concentration were investigated. Using these findings, the thermal activation process of dislocation motion during creep was characterized in an $\mathrm{Al}-\mathrm{Mg}$ solid-solution alloy.

\section{Experimental Procedures}

Ingots of $\mathrm{Al}-0.5 \mathrm{~mol} \% \mathrm{Mg}(\mathrm{Al}-0.5 \mathrm{Mg}), \mathrm{Al}-1.0 \mathrm{~mol} \% \mathrm{Mg}$ (Al-1.0Mg), Al-2.0 mol\% $\mathrm{Mg} \quad(\mathrm{Al}-2.0 \mathrm{Mg})$, and $\mathrm{Al}-$ $4.2 \mathrm{~mol} \% \mathrm{Mg}$ (Al-4.2Mg) alloys were purchased from Kojundo Chemical Laboratory Co. Ltd. They were homogenized in a muffle furnace under argon gas at $773 \mathrm{~K}$ for $24 \mathrm{~h}$. Then, they were cut into dimensions of approximately $5 \mathrm{~mm} \times 5 \mathrm{~mm} \times 3 \mathrm{~mm}$ by a cut-off machine. A test surface with dimensions of $5 \mathrm{~mm} \times 5 \mathrm{~mm}$ was polished using \#1200, \#2400 and \#4000 emery papers. The cut specimens were then annealed using a lamp thermal image furnace in argon gas at $773 \mathrm{~K}$ for $6 \mathrm{~h}$. Before the mechanical test, the surface oxide layer was immediately removed through electropolishing. The grains of each sample were equiaxed with an average grain size of approximately $100-300 \mu \mathrm{m}$.

The indentation tests were performed using a microindenter (Advance Riko Co.), equipped with a diamond conical indenter with a half-apex angle of $68^{\circ}$. Constantindentation creep rate tests were performed with the load controlled by a function $F=F_{0} \exp (2 \lambda t),{ }^{18)}$ where $F_{0}=$ $4.91 \times 10^{-2} \mathrm{~N}$ and $\lambda$ is the deformation rate, i.e., $\lambda=1 \times$ $10^{-5}-4 \times 10^{-3} \mathrm{~s}^{-1}$. When the load is controlled by the function and a specific deformation condition under the indenter is achieved, creep rate (indentation creep rate) and stress (indentation pressure) are kept constant. ${ }^{19,20)}$ By selecting various $\lambda$ values, different creep rates can be tested. Here, after reaching a load of $2.94 \mathrm{~N}$, the load was immediately increased to $3.14 \mathrm{~N}$ within $0.1 \mathrm{~s}$ to perform abrupt load change tests. The above tests were conducted at a test temperature of $673 \mathrm{~K}$ in a $99.9999 \%$ argon gas atmosphere. The data sampling rate was 100 points/s. The evaluation method of the effective stress and activation volume are described in further detail in Appendix A2.

\section{Evaluation Equations for the Internal Stress and Activation Volume}

The indentation creep rate $\dot{\varepsilon}_{\text {in }}$ and indentation pressure $p$ are defined by eqs. (1) and (2), respectively, based on the indenter displacement $u$ and indentation load $F:^{18-23)}$

$$
\begin{aligned}
& \dot{\varepsilon}_{\text {in }}=\frac{\dot{u}}{u}, \\
& p=\frac{F}{\pi u^{2} \tan \theta},
\end{aligned}
$$

where $\dot{u}$ is the indenter velocity, and $\theta$ is the half apex angle of the conical indenter. Under the indenter, various creep rates $\dot{\varepsilon}_{\mathrm{c}}$ and flow stresses $\sigma_{\mathrm{f}}$ occur. The deformation under the indenter is represented by a set of values given by $\dot{\varepsilon}_{\text {in }}$ and $p$. The uniaxial creep data of $\dot{\varepsilon}_{\mathrm{c}}$ and $\sigma_{\mathrm{f}}$ are related to $\dot{\varepsilon}_{\text {in }}$ and $p$ by: ${ }^{18-21)}$

$$
\begin{aligned}
& \dot{\varepsilon}_{\mathrm{c}} \cong C_{2} \dot{\varepsilon}_{\text {in }}=\dot{\varepsilon}, \\
& \sigma_{\mathrm{f}} \cong C_{1} p=\sigma,
\end{aligned}
$$

where $C_{1}$ is a constant referred to as the Tabor coefficient, ${ }^{21)}$ and $C_{2}$ is a constant reported in our previous papers. ${ }^{19,20)}$ In this study, $C_{1}=0.33^{21)}$ and $C_{2}=0.20^{20)}$ were used.

\subsection{Internal stress}

For the dislocations to move in metals and alloys, they need to overcome the thermal and athermal obstacles using the effective stress $\sigma_{\mathrm{e}}$ with thermal energy and internal stress $\sigma_{\mathrm{i}}$. The sum of $\sigma_{\mathrm{e}}$ and $\sigma_{\mathrm{i}}$ is the applied stress $\sigma$ of the mechanical test. When the shear plastic strain rate can be determined by the mobility of dislocation, an equation of the strain rate is given as follows, ${ }^{2)}$

$$
\dot{\gamma}=\rho_{\mathrm{m}} b \bar{v},
$$

where $\rho_{\mathrm{m}}$ is the mobile dislocation density, $b$ is the magnitude of the Burgers vector, and $\bar{v}$ is the average dislocation velocity. When $v=B \tau_{\mathrm{e}}{ }^{2,16,17,24)}$ the strain rate is

$$
\dot{\varepsilon}=\frac{1}{M^{2}} \rho_{\mathrm{m}} b B \sigma_{\mathrm{e}},
$$

where $M$ is the Taylor factor, and $B$ is the dislocation mobility.

Assuming that the dislocation substructure does not change after the abrupt stress change, the difference in strain rate is

$$
\dot{\varepsilon}_{2}-\dot{\varepsilon}_{1}=\frac{\rho_{\mathrm{m}} b B}{M^{2}}\left(\sigma_{\mathrm{e} 2}-\sigma_{\mathrm{e} 1}\right)
$$

where subscripts 1 and 2 denote before and after the abrupt stress change, respectively. Since the internal stress does not change, the effective stress in eqs. (6) and (7) can be replaced with the applied stress. Therefore, the equation for determining the internal stress is

$$
\sigma_{\mathrm{i} 1}=\sigma_{1}-\dot{\varepsilon}_{1}\left(\frac{\sigma_{2}-\sigma_{1}}{\dot{\varepsilon}_{2}-\dot{\varepsilon}_{1}}\right) .
$$

The effective stress $\sigma_{\mathrm{e} 1}$ can be derived by using $\sigma_{1}-\sigma_{\mathrm{i} 1}$.

\subsection{Activation volume}

The activation volume is related to the area swept by a certain dislocation length when the dislocation attempts to overcome the thermal obstacle using the effective stress and thermal energy. When the dislocation overcomes the thermal obstacle with some frequency by the thermal energy and effective stress, the dislocation velocity can be expressed as follows: $:^{3,4)}$

$$
v=v_{0} \exp \left(-\frac{\Delta G_{0}-\tau_{\mathrm{e}} V}{k T}\right)
$$

where $v_{0}$ is a constant, $\Delta G_{0}$ is the thermal energy required to overcome the thermal obstacle without effective stress, $V$ is the activation volume, and $k$ is the Boltzmann constant. The shear plastic strain rate in eq. (5) can then be expressed as: 


$$
\dot{\gamma}=\rho_{m} b v_{0} \exp \left(-\frac{\Delta G}{k T}\right)=\dot{r}_{0} \exp \left(-\frac{\Delta G}{k T}\right),
$$

where $\dot{\gamma}_{0}$ is a constant determined by the dislocation substructure.

If $\dot{\gamma}_{0}$ does not change after the abrupt stress change, the activation volume is

$$
V=M k T\left(\frac{\ln \left(\frac{\dot{\varepsilon}_{2}}{\dot{\varepsilon}_{1}}\right)}{\sigma_{2}-\sigma_{1}}\right) .
$$

\section{Results}

\subsection{Indentation creep data}

Figure 1 shows the time dependence of the indenter displacement $u$ in Al-Mg alloys at $T=673 \mathrm{~K}$ and $\lambda=1 \times$ $10^{-3} \mathrm{~s}^{-1}$. As shown in Fig. A1.1 of Appendix A1, the indentation creep rate $\dot{\varepsilon}_{\text {in }}$ and indentation pressure $p$ obtained from the data in Fig. 1 remained at certain constant values $\dot{\varepsilon}_{\text {in(s) }}(\cong \lambda)$ and $p_{\mathrm{s}}$. This finding indicates that pseudo-steady creep occurred under the indenter. ${ }^{19,20)}$ As shown in Fig. 1, the displacement of $\mathrm{Al}-0.5 \mathrm{Mg}$ was the largest, while that of $\mathrm{Al}-4.2 \mathrm{Mg}$ was the smallest, even though the indentation load was the same with respect to indentation time. This result indicates that creep is more difficult in an alloy with higher solute concentration, i.e., $\mathrm{Al}-4.2 \mathrm{Mg}$.

Figure 2 shows the time dependence of the indenter displacement when the load was increased from $F_{1}=2.94 \mathrm{~N}$ to $F_{2}=3.14 \mathrm{~N}$ for $\mathrm{Al}-2.0 \mathrm{Mg}$. The load was changed when the indentation creep rate and indentation pressure became constant at $\dot{\varepsilon}_{\text {in(s) }}(\cong \lambda)$ and $p_{\mathrm{s}}$, as shown in Fig. A1.1 of Appendix A1. The increase in creep rate (stress) before and after the abrupt load change can be obtained using $\dot{\varepsilon}_{1} \propto \lambda$, $\sigma_{1} \propto F_{1} / u_{1}^{2}\left(\propto p_{\mathrm{s}}\right)$ and $\dot{\varepsilon}_{2} \propto \frac{\dot{u}_{2}}{u_{2}}, \sigma_{2} \propto F_{2} / u_{2}^{2}$, respectively. As shown in Fig. 2, $\dot{u}_{2}$ was obtained by a linear approximation of the experimental data after the load increase. The internal stress and activation volume can be obtained by substituting these experimental values into eqs. (8) and (11), respectively. These values must be measured when the dislocation substructure does not change. In this study, the absence of

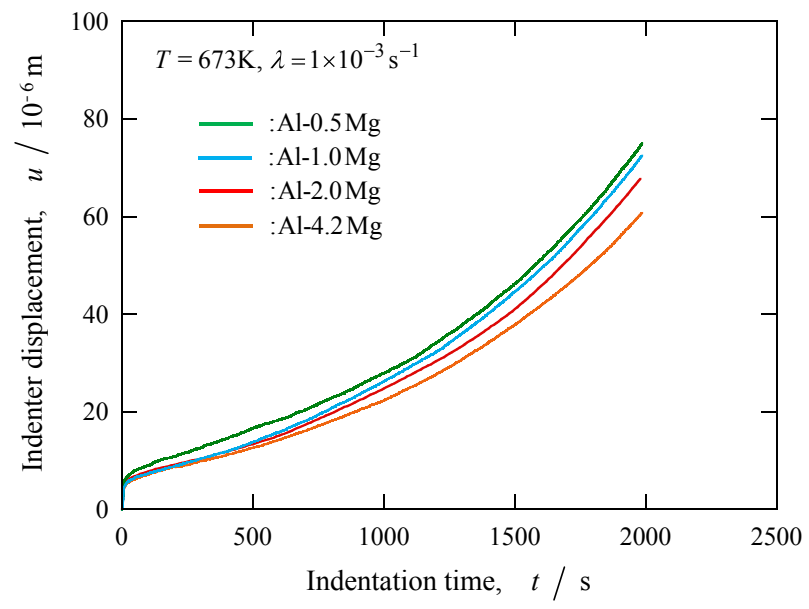

Fig. 1 Indentation creep curves of $\mathrm{Al}-0.5 \mathrm{Mg}, \mathrm{Al}-1.0 \mathrm{Mg}, \mathrm{Al}-2.0 \mathrm{Mg}$, and Al-4.2Mg.

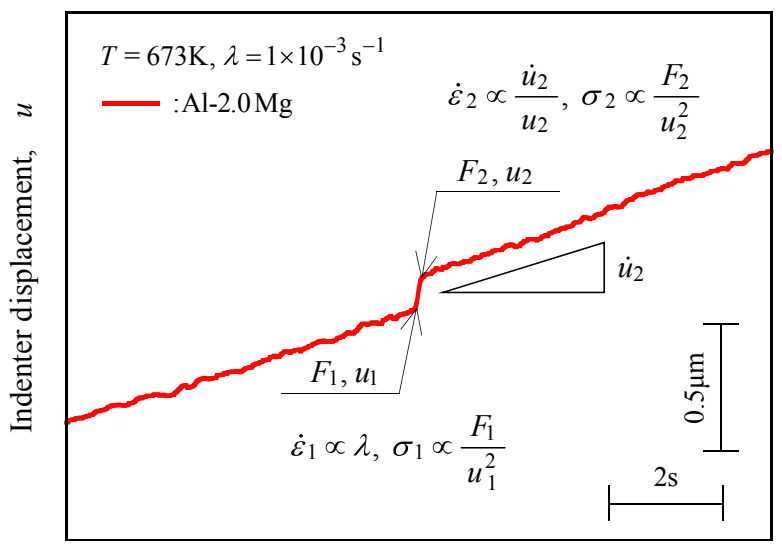

Indentation time, $t$

Fig. 2 Time dependence of the indenter displacement when the load was abruptly increased from $F_{1}=2.94 \mathrm{~N}$ to $F_{2}=3.14 \mathrm{~N}$.

an instantaneous plastic displacement when the load abruptly increased was confirmed. However, creep actually occurs in the range of the linear approximation used to obtain the $\dot{u}_{2}$-value, so this range of the linear approximation should be as short as possible. Nonetheless, as shown in Fig. A2.1, changing this range did not affect the obtained activation volumes, which suggests that the above-mentioned experimental values were appropriate, as explained in further detail explanation in Appendices A2 and A3.

\subsection{Internal stress}

Figure 3 shows the ratio of the internal stress $\sigma_{i 1}$ to the applied stress $\sigma_{1}$ for $\mathrm{Al}-4.2 \mathrm{Mg}$. The values of $\sigma_{\mathrm{i} 1} / \sigma_{1}$, which decreased from 0.85 to 0.64 with increasing stress, were obtained from experimental data similar to those presented in Fig. 2 and eq. (8). The black line shows the results of the tensile test for $\mathrm{Al}-5.7 \mathrm{Mg}{ }^{25)}$ The absolute values are different due to the difference in solute concentration; however, the tendency in the stress dependence is similar. The results for the other solute concentrations obtained in this experiment at $T=673 \mathrm{~K}$ were as follows: $\sigma_{\mathrm{i} 1} / \sigma_{1}=0.85-0.77, \sigma_{\mathrm{i} 1} / \sigma_{1}=$ $0.88-0.75$, and $\sigma_{\mathrm{i} 1} / \sigma_{1}=0.80-0.69$ for $\mathrm{Al}-0.5 \mathrm{Mg}$, Al$1.0 \mathrm{Mg}$, and $\mathrm{Al}-2.0 \mathrm{Mg}$, respectively.

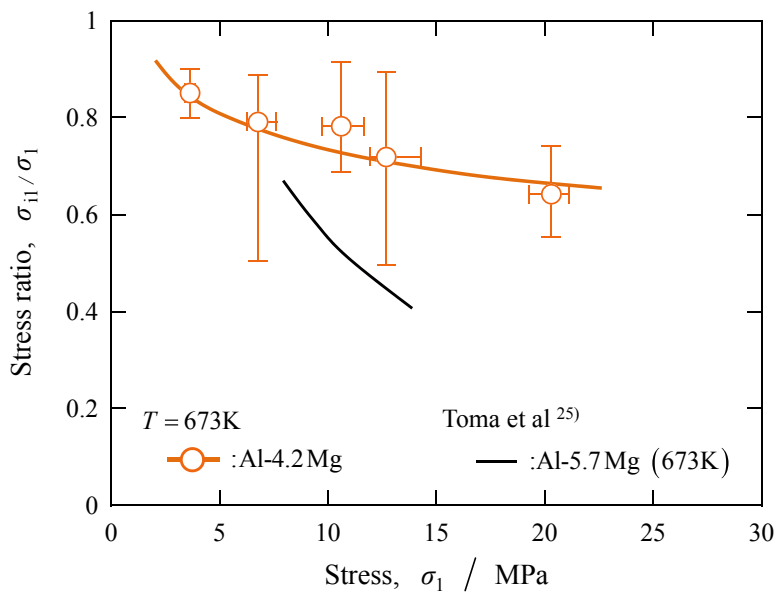

Fig. 3 Ratio of the internal stress $\sigma_{\mathrm{i} 1}$ to the applied stress $\sigma_{1}\left(\sigma_{\mathrm{i} 1} / \sigma_{1}\right)$ for Al4.2Mg. The black curve shows the tensile test results for $\mathrm{Al}-5.7 \mathrm{Mg}$. ${ }^{25}$ ) 


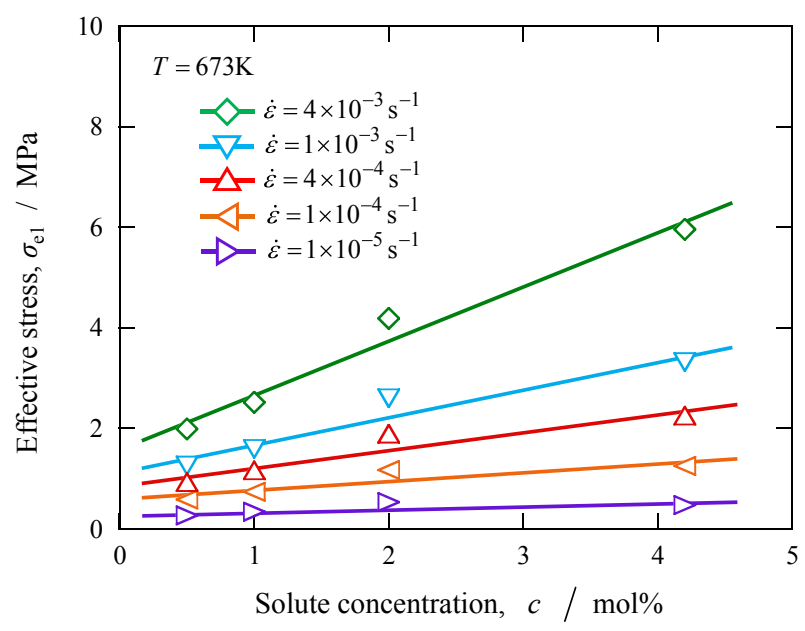

Fig. 4 Relation between the solute concentration and the effective stress $\sigma_{\mathrm{e} 1}$.

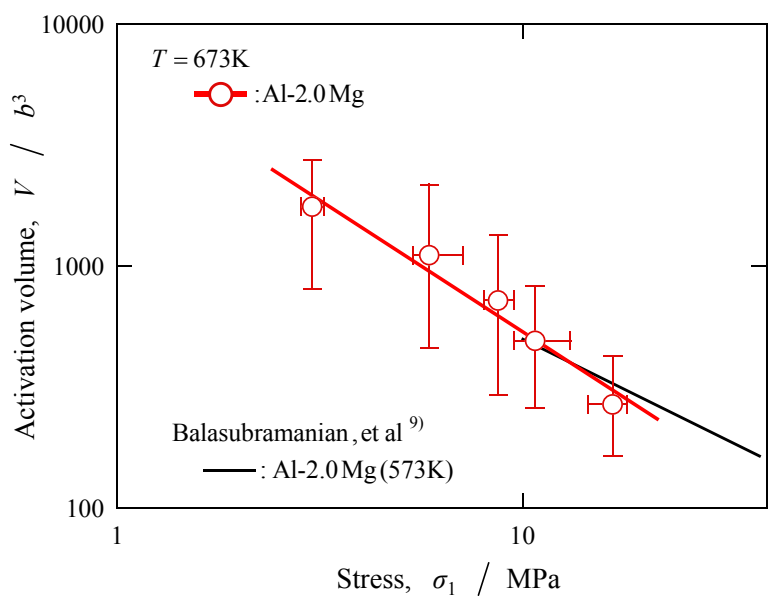

Fig. 5 Stress dependence of the activation volume $V$ for $\mathrm{Al}-2.0 \mathrm{Mg}$. The black line indicates the uniaxial test results. ${ }^{9}$ )

Figure 4 shows the relationship between the solute concentration and the effective stress $\sigma_{\mathrm{e} 1}$. This graph depicts only average values. At each particular creep rate, the effective stress increased with increasing solute concentration. Additionally, at each solute concentration, the ratio increased with increasing creep rate; this trend is particularly visible for higher solute concentrations. The results suggest that the effective stress for overcoming the thermal obstacles, e.g., solute atmosphere, exists, implying that the dislocations in $\mathrm{Al}-\mathrm{Mg}$ alloys move viscously. ${ }^{2,16,17)}$

\subsection{Activation volume}

Figure 5 shows the stress dependence of the activation volume $V$ for $\mathrm{Al}-2.0 \mathrm{Mg}$, obtained from experimental data similar to that in Fig. 2 and using eq. (11). The obtained values were $V=2300 b^{3}-270 b^{3}$ in $\sigma_{1}=3.0-16.6 \mathrm{MPa}$, where $b=2.86 \times 10^{-10} \mathrm{~m}$. The black line in the figure indicates the results of the uniaxial test ${ }^{9}{ }^{9}$ the obtained results correspond well with the absolute values and stress dependence from the literature.

Figure 6 shows the relationship between solute concentration and the activation volume $V$. The data are only average values, which have similar deviations, as shown in Fig. 5. At each constant creep rate, the $V$ values decreased as the solute

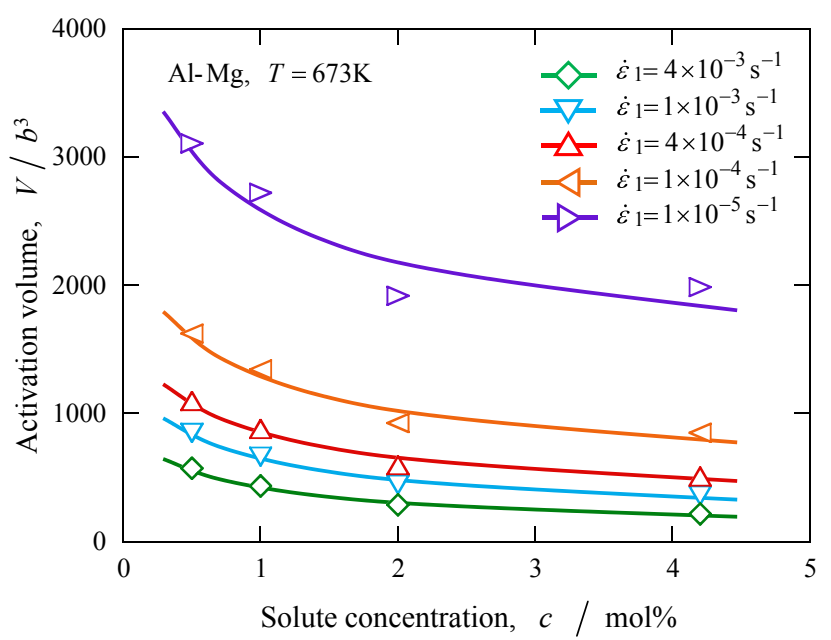

Fig. 6 Relation between the solute concentration $c$ and activation volume

concentration increased. Additionally, at each solute concentration, the ratio decreased with the increasing creep rate. The dependences of the solute concentration and creep rate on the obtained activation volume thus have an opposite tendency to that of effective stress (Fig. 4), which implies that the activation volume can be used to characterize the dislocation motion.

\section{Discussions}

The reason for the reduction in activation volume with increasing solute concentration and creep rate (Fig. 6) is as follows. When the solute concentration becomes relatively high, dislocation motion requires higher levels of effective stress, ${ }^{2,16,17)}$ implying the presence of more thermal obstacles i.e., solute atoms interact with moving dislocations. In addition, when the creep rate increases, the thermal energy required for dislocation motion decreases, implying that the thermal activation process of dislocation motion, i.e., the length and distance shift, depends on the creep rate and solute concentration. Therefore, the activation volume can be used to characterize the dislocation motion during creep. The following section discusses the thermally activated dislocation length and distance in the case of interactions between dislocations and solute atoms.

The activation volume is defined as the swept area $l w$ when a dislocation length of $l$ overcomes the thermal obstacle and moves by distance $w$. Hence, to characterize the thermally activated process on the dislocation in the creep, the length $l$ and distance $w$ should be determined.

One of the possible lengths for the thermally activated process is the length between forest dislocations. From the dislocation density $\rho$, the above length $l$ can be determined by $l=1 / \sqrt{\rho}$. From the transmission electron microscopy (TEM) observations, the dislocation density in Al-Mg alloys was determined to be $\rho \cong 10^{12} \mathrm{~m}^{-2}$ at a flow stress of $\sim 10 \mathrm{MPa}{ }^{26,27)}$ From the dislocation density, the length $l$ $(=1 / \sqrt{\rho})$ was calculated to be $l \cong 10^{-6} \mathrm{~m}$. When the entire dislocation, where $l \cong 10^{-6} \mathrm{~m}$, moved via a thermally activated process, the distance $w(=V / l b)$ was $w \cong 4.2 \times$ $10^{-11} \mathrm{~m}$, as determined using the activation volume data 
$\left(V=512 b^{3}\right)$ in $\mathrm{Al}-4.2 \mathrm{Mg}$ at $10 \mathrm{MPa}$. The $w$ value was significantly smaller than the magnitude of the Burgers vector $b=2.86 \times 10^{-10} \mathrm{~m}$, which means that the moved dislocations did not encounter any thermal obstacles. However, considering the thermal activation process of the dislocation motion during creep, the $w$ value mentioned above is unacceptably small. The cause of this underestimate may be that the thermally activated dislocation length $l$ was set as the length between forest dislocations, i.e., $l$ was overestimated.

To estimate a reasonable combination of $l$ and $w$, the computer simulation results for the interaction between dislocations and solute atoms in solid-solution alloys of Leyson et al. ${ }^{12-14)}$ were used. Numerical calculations were performed based on the dislocation motion model proposed by Labusch, ${ }^{15)}$ with the assumption that moving dislocations interact simultaneously with several solute atoms. The results show that for the thermally activated dislocation length $l$ and distance $w$, values of $l_{\mathrm{c}}$ and $w_{\mathrm{c}}$ exist for which the total dislocation energy is minimized. ${ }^{12,13)}$ Moreover, $l$ and $w$ increase with increasing temperature. The relationship among $l, w, l_{\mathrm{c}}$, and $w_{\mathrm{c}}$ is then

$$
\frac{l}{l_{\mathrm{c}}}=\left(\frac{w}{w_{\mathrm{c}}}\right)^{\phi},
$$

where $\phi=1.47 .{ }^{14)}$ Through molecular dynamics simulations using EAM potentials in the context of the Labusch model, Olmsted et al. obtained two possible cases of $l_{\mathrm{c}}$ and $w_{\mathrm{c}}$, namely, $l_{\mathrm{c}} \cong 32 \times 10^{-10} \mathrm{~m}$ and $w_{\mathrm{c}} \cong 10 \times 10^{-10} \mathrm{~m}$ at absolute zero (case 1 ) and $l_{\mathrm{c}} \cong 52 \times 10^{-10} \mathrm{~m}$ and $w_{\mathrm{c}} \cong 20 \times$ $10^{-10} \mathrm{~m}$ at $300 \mathrm{~K}$ (case 2 ), respectively, for edge dislocations in $\mathrm{Al}-5 \mathrm{~mol} \% \mathrm{Mg} .{ }^{28)}$ By substituting these values of $l_{\mathrm{c}}, w_{\mathrm{c}}$, and $\phi$ into eq. (12), the following relationships are obtained:

$$
\begin{gathered}
\frac{l}{w^{1.47}} \cong 54344\left(=\frac{l_{\mathrm{c}}}{w_{\mathrm{c}}^{1.47}}\right) \quad \text { for case } 1 \\
\frac{l}{w^{1.47}} \cong 31878\left(=\frac{l_{\mathrm{c}}}{w_{\mathrm{c}}^{1.47}}\right) \text { for case } 2 .
\end{gathered}
$$

The combination of $l_{\text {cal }}(=l)$ and $w_{\text {cal }}(=w)$ in eqs. (13) and (14) is determined when these two equations hold. The calculated activation volume $V_{\text {cal }}\left(=l_{\text {cal }} w_{\text {cal }} b\right)$ is associated with the activation volume of $\mathrm{Al}-4.2 \mathrm{Mg}$ determined in this study. Figure 7 shows the stress dependences of $l_{\text {cal }}$ and $w_{\text {cal }}$ obtained from the activation volume in $\mathrm{Al}-4.2 \mathrm{Mg}$ and from eqs. (13) and (14). In the figure, the blue lines represent eq. (13), and the values are $l_{\text {cal }}=3.0 \times 10^{-8}-7.2 \times 10^{-9} \mathrm{~m}$ and $w_{\text {cal }}=4.4 \times 10^{-9}-1.7 \times 10^{-9} \mathrm{~m}$ at $\sigma=3.8-20 \mathrm{MPa}$. The red lines represent eq. (14), and the values are $l_{\text {cal }}=2.4 \times$ $10^{-8}-5.8 \times 10^{-9} \mathrm{~m}$ and $w_{\text {cal }}=5.6 \times 10^{-9}-2.1 \times 10^{-9} \mathrm{~m}$. The swept area $\left(l_{\text {cal }} w_{\text {cal }}\right)$ was about $1.3 \times 10^{-16}-1.2 \times$ $10^{-17} \mathrm{~m}^{2}$ in the case illustrated in Fig. 7. Thus, the $l_{\text {cal }} w_{\text {cal }}$ value was $150-1500$ times greater than $b^{2}(b=2.86 \times$ $\left.10^{-10} \mathrm{~m}\right)$. In other words, about 6-60 solute atoms were present in the swept area, which implies that the moving dislocations interacted simultaneously with several solute atoms. This is consistent with the conditions of the simulation, from which eq. (12) was derived. Additionally, Marukawa postulated a version of the plural-obstacle theory for solution-hardening in fcc metals, for the case of dislocation controlled by thermally activated processes. ${ }^{29)}$ For interactions between dislocations and plural solute atoms

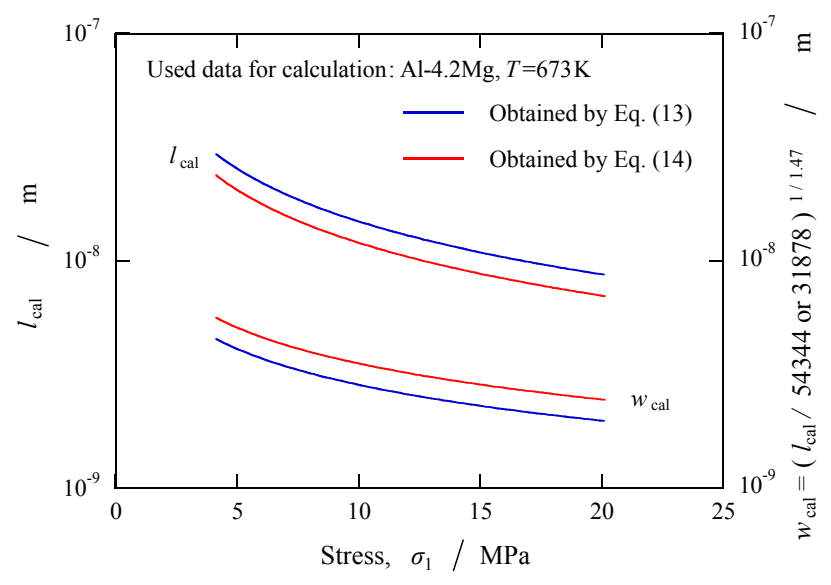

Fig. 7 Stress dependence of $l_{\text {cal }}$ and $w_{\text {cal }}$, which were determined using experimental data of the activation volume obtained in this study and computer simulation results reported elsewhere. ${ }^{12-14)}$

during a single activation event, the activation volume was shown to depend on the temperature and solute concentration, and the calculated activation volume was shown to reduce with increasing solute concentration. These findings support the results of this study. Therefore, the $l_{\text {cal }}$ and $w_{\text {cal }}$ obtained via the above-mentioned process, as shown in Fig. 7, can then be considered the thermally activated dislocation length and distance during creep, respectively. However, to check the validity of the estimated values of the $l_{\text {cal }}$ and $w_{\text {cal }}$, in situ TEM observations are needed. Further discussion of the validity will be reported elsewhere.

Without confirming the validity of these values of $l_{\text {cal }}$ and $w_{\text {cal }}$, the following aspects can be addressed. As shown in Fig. 7, the stress dependence of the obtained activation volume can be explained by stating that the thermally activated dislocation length and distance decreased with increasing stress. Further, with reference to Fig. 6, the dislocation length and distance actually decreased with both increasing solute concentration and increasing creep rate.

The above discussion of the thermally activated dislocation length and distance was based on the theory and simulation results from observing the interactions between a dislocation and several solute atoms. In contrast, during creep in $\mathrm{Al}-\mathrm{Mg}$ solid-solution alloys, dislocations reportedly move with the solute atmosphere. ${ }^{2,16,17)}$ The idea of viscous motion for interactions with solute atoms differs from that of dragging with a solute atmosphere. However, the abovementioned dislocation motions exhibited a similar behavior, i.e., viscous motion. According to previous TEM observations, dislocations in the $\mathrm{Al}-\mathrm{Mg}$ alloy are distributed randomly and have many bending parts. ${ }^{27)}$ Additionally, the dislocations bow out at several places onto the long dislocation length, which is the length between forest dislocations, corresponding to the discussion of Fig. 7 above. Therefore, the obtained results shown in Fig. 7 can be used to characterize the dislocation motion during creep.

\section{Conclusions}

(1) The obtained activation volume and existing effective stress in $\mathrm{Al}-\mathrm{Mg}$ solid-solution alloys were consistent 
with those obtained by using uniaxial tests. Therefore, indentation techniques could be used in evaluating the thermal activation parameters.

(2) At a constant creep rate, the activation volume decreased with the increasing solute concentration, and it increased with the increasing temperature in $\mathrm{Al}-$ $\mathrm{Mg}$ solid solution alloys. The opposite tendencies were observed for the effective stress when the corresponding effective stress was interpolated against the increase/ decrease in the thermally activated dislocation length, which suggests that during thermal activation, the dislocations could overcome thermal obstacles.

(3) When the thermally activated dislocation length was assumed to be equal to the length between forest dislocations, the activated distance was much smaller than the magnitude of the Burgers vector. In contrast, when the length was set to that obtained from previously reported computer simulation results, the calculated distance was found to be appropriate. The calculated results suggest that each dislocation fragment on the length between forest dislocations moved individually through each thermal activation process.

The above results demonstrate that the activation volume, including the effective stress, can be used to characterize the thermal activation process of dislocation motion. In consequence, the obtained results eventually help to propose several ways to improve the creep properties, such as creep strength and resistance. However, it remains necessary to observe the dislocation structure, such as the thermally activated length, by using TEM, in order to validate the relationship between the activation volume and the activated dislocation length and distance. Additionally, the abovementioned treatment should also be conducted for different solid-solution alloys, which have different mobilities for their solute atoms and different crystal structures.

\section{Acknowledgement}

This study was supported by the Light Metal Education Foundation of Japan. The indentation tests were performed with the cooperation of Hitoshi Igarashi and Ryutaro Iimura, graduate students of the Graduate School of Engineering, Nihon University.

\section{Appendix}

\section{A1 Indentation Data for Evaluating Creep Parameters}

Figure A1.1 shows the time dependence of the indentation creep rate $\dot{\varepsilon}_{\text {in }}$ and indentation pressure $p$ obtained from Fig. 1 . In this figure, these values asymptotically approach certain constant values $\dot{\varepsilon}_{\text {in(s) }}$ and $p_{\mathrm{s}}$, which are the pseudo-steady state properties realized under the indenter. ${ }^{19,20)}$ In addition, $\dot{\varepsilon}_{\text {in(s) }}$ is independent of the specimen and is equal to the $\lambda$ value of the load function $F=F_{0} \exp (2 \lambda t)$. As shown in Fig. A1.1, individual sets of constant values were obtained from the experimental data each set of conditions tested in this study.

Using the above data for $\dot{\varepsilon}_{\text {in(s) }}$ and $p_{\mathrm{s}}$, the stress exponent for creep can be obtained. Figure A1.2 shows a doublelogarithmic plot of $\dot{\varepsilon}_{\mathrm{s}}\left(=C_{2} \dot{\varepsilon}_{\text {in(s) }}\right)$ and $\sigma_{\mathrm{s}}\left(=C_{1} p_{\mathrm{s}}\right)$. The

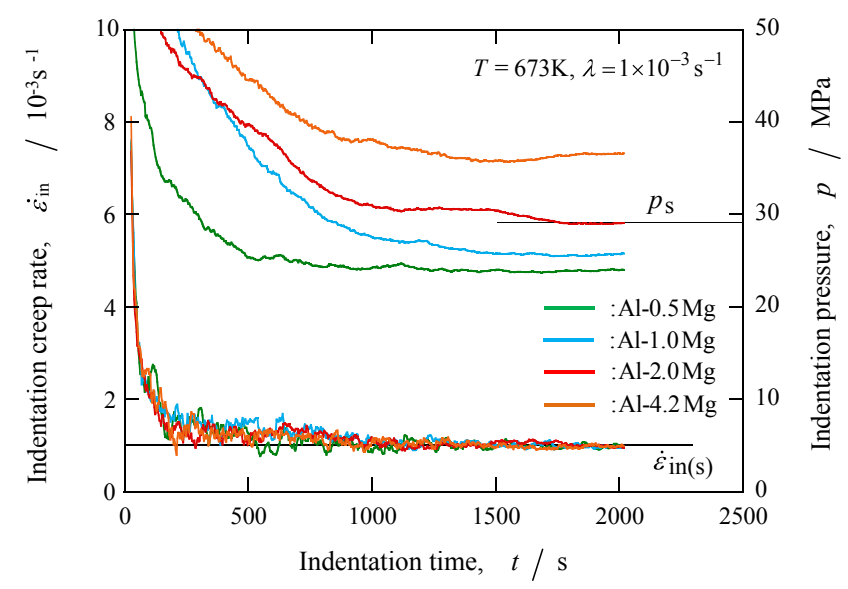

Fig. A1.1 Indentation time variations of the indentation creep rate $\dot{\varepsilon}_{\text {in }}$ and indentation pressure $p$ obtained from Fig. 1. After a certain indention time, these curves become constant with $\dot{\varepsilon}_{\text {in(s) }}$ and $p_{\mathrm{s}}$.

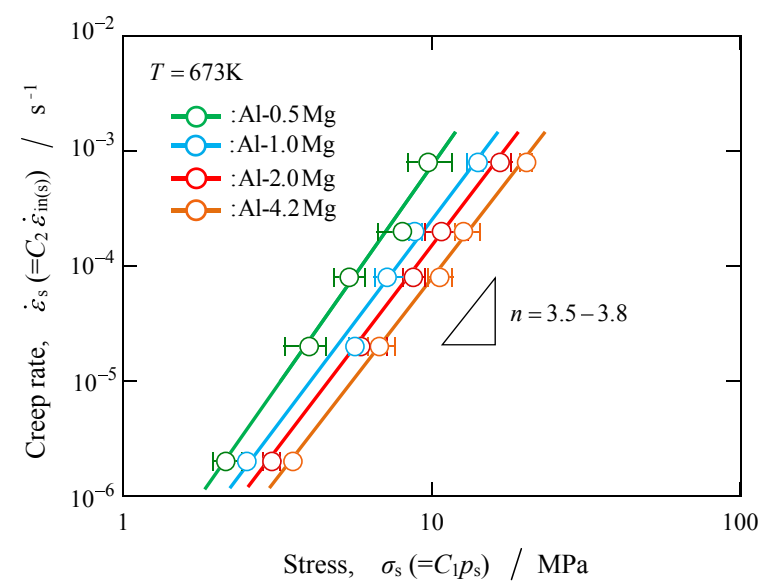

Fig. A1.2 Double-logarithmic plot of $\dot{\varepsilon}_{\mathrm{s}}\left(=C_{2} \dot{\varepsilon}_{\text {in(s) }}\right)$ and $\sigma_{\mathrm{s}}\left(=C_{1} p_{\mathrm{s}}\right)$. The slopes of these plots correspond to the stress exponent $n$.

slopes of these straight lines correspond to the stress exponent $n$. At $T=673 \mathrm{~K}, n=3.8,3.6,3.6$, and 3.5 for $\mathrm{Al}-0.5 \mathrm{Mg}, \mathrm{Al}-1.0 \mathrm{Mg}, \mathrm{Al}-2.0 \mathrm{Mg}$, and $\mathrm{Al}-4.2 \mathrm{Mg}$, respectively. These results correspond to those obtained from the creep tests reported by other researchers: $n=3.2$ at $T=$ $600 \mathrm{~K}$ for $\mathrm{Al}-0.5 \mathrm{Mg}^{30)} n=3.3$ and 3.5 at $T=600$ and $640 \mathrm{~K}$, respectively, for $\mathrm{Al}-1.0 \mathrm{Mg}^{30)} n=3.1$ at $T=600 \mathrm{~K}$ for $\mathrm{Al}-3.0 \mathrm{Mg},{ }^{30)}$ and $n=2.8-3.0$ at $T=601-734 \mathrm{~K}$ for Al$5.1 \mathrm{Mg} .{ }^{31)}$ The obtained $n$-values are close to $n=3$, in good agreement with our previously reported results. ${ }^{19,20)}$ In addition, under the testing conditions in previous studies, $2,16,17,19,20,30-32)$ the dislocations exhibited viscous glide, and the creep rate was controlled by the dislocation motion.

Using the present testing method, the data for evaluating the stress exponent, internal stress, and activation volume could be acquired from a single indentation test.

\section{A2 Method for Determining the Indenter Velocity after Load Increase}

To determine the internal stress and activation volume, it is necessary to know the creep rate before and after the abrupt load increase. As explained in the "Internal stress and activation volume" section, the deformation rate before the abrupt load increase can be obtained from $\dot{\varepsilon}_{1} \propto \lambda$. In contrast, 
$\dot{\varepsilon}_{2}$ is determined from $\frac{\dot{u}_{2}}{u_{2}}$ in eq. (1), where $\dot{u}_{2}$ is obtained through a linear approximation of the indenter displacement after the abrupt load increase. Here, it is necessary to determine the data range that should be approximated. If the approximation range is widened, there is a high possibility of changing the dislocation substructure under the indenter. In contrast, when the approximation range is narrowed, the number of approximated data points decreases, resulting in a larger deviation from the obtained values. In addition, since the amount of indenter displacement depends on the test conditions, such as the deformation rate and temperature, it is difficult to determine the approximate range by the increment of time or indenter displacement. Therefore, in this study, an indentation strain increment was defined as follows.

Under the indenter, stress gradients are generated, and corresponding strain gradients are also formed. Hence, it is difficult to uniquely define an indentation strain. Herein, we used the equation for the indentation strain increment derived by definitely integrating both sides of eq. (1) over a certain range:

$$
\Delta \varepsilon=\varepsilon_{3}-\varepsilon_{2}=\frac{\ln u_{3}}{\ln u_{2}},
$$

where $u_{3}$ is an indenter displacement for a certain time point after the abrupt load increase. Furthermore, we did not learn the physical meaning of the indentation strain increment described in eq. (A1).

If $\Delta \varepsilon$ is determined, $u_{3}$ can be determined by eq. (A1). Figure A2.1 shows the activation volume when $\Delta \varepsilon$ is set to $0.002-0.01$ using the experimental data at $T=673 \mathrm{~K}$ for Al$2.0 \mathrm{Mg}$ shown in Fig. 5. The approximation range changes depending on $\Delta \varepsilon$, resulting in variations in $\dot{\varepsilon}_{2}=\frac{C_{2} \dot{u}_{2}}{u_{2}}$. The $\dot{\varepsilon}_{1}$, $\sigma_{1}$, and $\sigma_{2}$ values in Fig. 2 are not significantly different, and even $\Delta \varepsilon$ changes. When $\Delta \varepsilon$ is smaller, the deviation of the activation volume is slightly larger. However, there is no difference between the mean values. This tendency is similar to that for other samples and testing conditions. Additionally, the obtained internal stress did not depend on the value of $\Delta \varepsilon$. Considering these results, $\Delta \varepsilon=0.003$ was adopted. Using the obtained values of $\dot{\varepsilon}_{1}$ and $\dot{\varepsilon}_{2}, \sigma_{1}, \sigma_{2}$ in Fig. 2, the internal stress and activation volume were obtained using eqs. (8) and (11).

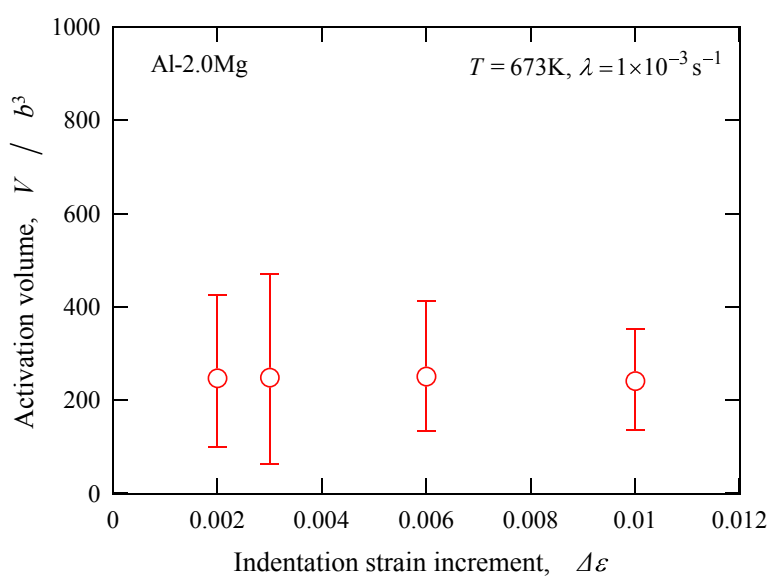

Fig. A2.1 Activation volume when $\Delta \varepsilon$ was set to 0.002 to 0.01 using the experimental data shown in Fig. 5.

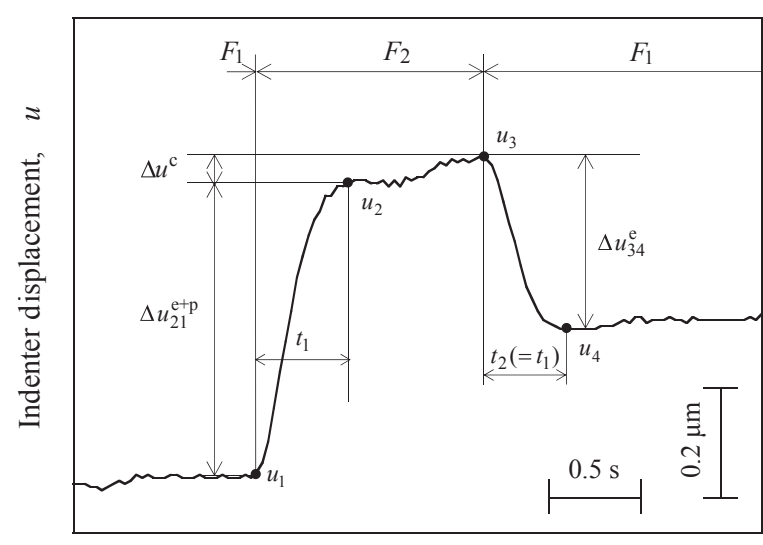

Indentation time, $t$

Fig. A3.1 Time dependence of indenter displacement, which significantly increased when the load was abruptly increased from $F_{1}$ to $F_{2}$.

\section{A3 Influence of Increment in Load on Evaluated Thermal Activation Parameters}

As the load abruptly increases in the indentation test, the stress under the indenter increases, and additional plastic deformation can occur under the indenter. When deriving the equations, it was assumed that the dislocation substructure remained constant. Therefore, it is necessary to confirm that there is no instantaneous plastic deformation within the displacement range of the detector, which was attached to the microindenter used in this study.

Figure A3.1 shows the time dependence of the indenter displacement when the load was abruptly increased from $F_{1}$ to $F_{2}$. After $0.3 \mathrm{~s}, F_{2}$ decreased back to the original load $F_{1}$. Here, the time for the load increase from $F_{1}$ to $F_{2}$ was similar to that in the abrupt load change tests in Fig. 2. As shown in Fig. A3.1, when the load was suddenly increased at a certain displacement, the indenter displacement became $u_{2}$ due to the elastic deformation of the measurement system and the elastic and instantaneous plastic deformation of the specimen. Subsequently, the displacement increased to $u_{3}$ due to the creep occurrence $\Delta u^{\mathrm{c}}$. When the load returned to the original value $F_{1}$, the indenter displacement became $u_{4}$ due to the elastic deformation of the measurement system and the specimen. Here, $\Delta u_{21}^{\mathrm{e}+\mathrm{p}}=u_{2}-u_{1}$ and $\Delta u_{34}^{\mathrm{e}}=u_{3}-u_{4}$. When $\Delta u_{21}^{\mathrm{e}+\mathrm{p}}>\Delta u_{34}^{\mathrm{e}}$, instantaneous plastic deformation occurred when the load abruptly increased. In contrast, when $\Delta u_{21}^{\mathrm{e}+\mathrm{p}} \cong \Delta u_{34}^{\mathrm{e}}$, instantaneous plastic deformation did not occur within the detection displacement range. Furthermore, $\Delta u^{\mathrm{c}}$ values were significantly smaller than $\Delta u_{21}^{\mathrm{e}+\mathrm{p}}$ and $\Delta u_{34}^{\mathrm{e}}$ for all testing conditions in this study.

Figure A3.2 shows the relationship between the instantaneous displacement $\Delta u_{21}^{\mathrm{e}+\mathrm{p}}$ or $\Delta u_{34}^{\mathrm{e}}$ and the stress $\sigma_{2}\left(=\sigma_{1}+\right.$ $\Delta \sigma)$ at $T=673 \mathrm{~K}$ in $\mathrm{Al}-2.0 \mathrm{Mg}$. When the deformation rate was low $\left(\lambda=1 \times 10^{-4} \mathrm{~s}^{-1}\right), \Delta u_{21}^{\mathrm{e}+\mathrm{p}} \cong \Delta u_{34}^{\mathrm{e}}$ held, even when $\Delta \sigma$ was larger. On the contrary, when the deformation rate was high $\left(\lambda=4 \times 10^{-3} \mathrm{~s}^{-1}\right), \Delta u_{21}^{\mathrm{e}+\mathrm{p}}>\Delta u_{34}^{\mathrm{e}}$ when $\Delta \sigma$ was higher. The arrows in Fig. A3.2 indicate the $\sigma_{1}$ and $\sigma_{2}$ values corresponding to the respective stress values during the abrupt load change tests, described in the "Internal stress and activation volume" section. As evident from Fig. A3.2, the abrupt load change tests were performed under $\Delta u_{21}^{\mathrm{e}+\mathrm{p}} \cong$ $\Delta u_{34}^{\mathrm{e}}$, i.e., no additional plastic deformation occurred. Thus, 


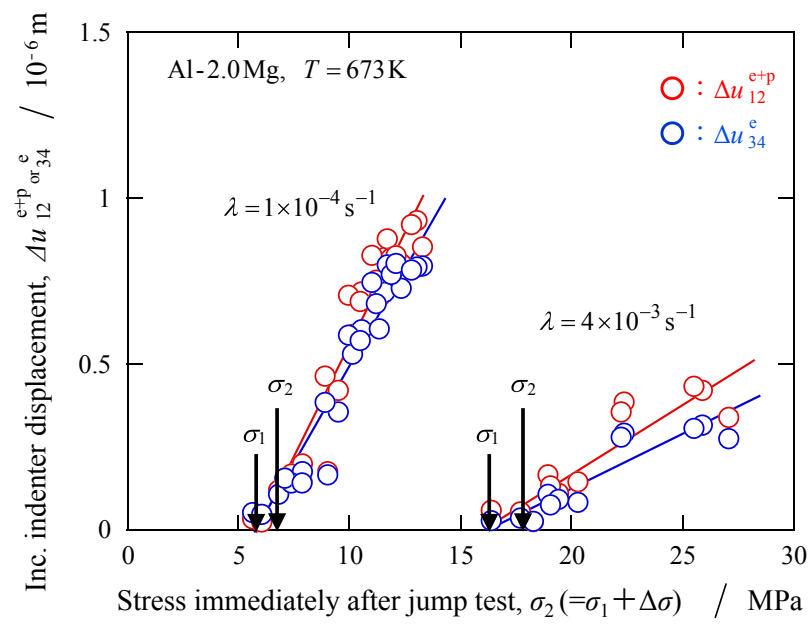

Fig. A3.2 Relationship between the instantaneous displacement $\Delta u_{21}^{\mathrm{e}+\mathrm{p}}$ or $\Delta u_{34}^{\mathrm{e}}$ and stress $\sigma_{2}\left(=\sigma_{1}+\Delta \sigma\right)$. The arrows indicate $\sigma_{1}$ and $\sigma_{2}$ values corresponding to the respective stress values, which occurred during the abrupt load change tests described in Section 4 and Appendix A2.

the dislocation substructure hardly changed. For other testing conditions, the abrupt load change tests were performed under the condition of $\Delta u_{21}^{\mathrm{e}+\mathrm{p}} \cong \Delta u_{34}^{\mathrm{e}}$.

\section{REFERENCES}

1) J. Cadek: Creep in Metallic Materials, (Elsevier, Amsterdam, 1988).

2) T. Suzuki, S. Takeuchi and H. Yoshinaga: Dislocation Dynamics and Plasticity, (Springer-Verlag, Berlin, 1991).

3) B.J. Diak, K.R. Upadhyaya and S. Saimoto: Prog. Mater. Sci. 43 (1998) 223-363.

4) D. Caillard and J.L. Martin (eds.): Thermally Activated Mechanisms in Crystal Plasticity, (Elsevier, Amsterdam, 2003).

5) I.-C. Choi, C. Brandl and R. Schwaiger: Acta Mater. 140 (2017) $107-$ 115.

6) M. Tanaka, D. Izumi, N. Yoshinura, G. Shigesato, M. Hoshino, K.
Ushioda and K. Higashida: Philos. Mag. 97 (2017) 2915-2930.

7) M.A. Meyers, A. Mishra and D.J. Benson: Prog. Mater. Sci. 51 (2006) 427-556.

8) G. Laplanche, J. Bonneville, C. Varvenne, W.A. Curtin and E.P. George: Acta Mater. 143 (2018) 257-264.

9) N. Balasubramanian and J.C.M. Li: J. Mater. Sci. 5 (1970) 434-444.

10) A. Orlova, J. Bonneville and P. Spatig: Mater. Sci. Eng. A 191 (1995) 85-89.

11) P. Zhang and Q.P. Kong: Philos. Mag. 79 (1999) 1-8.

12) G.P.M. Leyson, W.A. Curtin, L.G. Hector, Jr. and C.F. Woodward: Nat. Mater. 9 (2010) 750-755.

13) G.P.M. Leyson, L.G. Hector, Jr. and W.A. Curtin: Acta Mater. 60 (2012) 3873-3884

14) G.P.M. Leyson and W.A. Curtin: Model. Simul. Mater. Sci. Eng. 24 (2016) 065005.

15) R. Labusch: Phys. Status Solidi 41 (1970) 659-669.

16) H. Yoshinaga and S. Morozumi: Philos. Mag. 23 (1971) 1367-1385.

17) T. Endo, T. Shimada and T.G. Langdon: Acta Metall. 32 (1984) 19911999.

18) B.N. Lucas and W.C. Oliver: Metall. Mater. Trans. A 30 (1999) 601610.

19) H. Takagi, M. Dao and M. Fujiwara: Mater. Trans. 55 (2014) 275-284

20) H. Takagi and M. Fujiwara: Mater. Sci. Eng. A 602 (2014) 98-104.

21) D. Tabor: The Hardness of Metals, (Oxford University Press, Oxford, 1951).

22) P.J. Blau and B.R. Lawn: Microindentation Techniques in Materials Science and Engineering, (ASTM, Philadelphia, 1986).

23) P.M. Sargent and M.F. Ashby: Mater. Sci. Technol. 8 (1992) 594-601.

24) H. Cottrell and M.A. Jaswon: Proc. R. Soc. London, Ser. A 199 (1949) 104-114.

25) K. Toma, H. Yoshinaga and S. Morozumi: Mater. Trans. JIM 17 (1976) $102-110$.

26) H. Oikawa and A. Yasuda: Met. Sci. 13 (1979) 551-553.

27) H. Hayakawa, H. Nakashima and H. Yoshinaga: J. Japan Inst. Metals 53 (1989) 1113-1122.

28) D.L. Olmsted, L.G. Hector, Jr. and W.A. Curtin: J. Mechan. Phys. Solids 54 (2006) 1763-1788.

29) K. Marukawa: Philos. Mag. 87 (2007) 4027-4046.

30) H. Sato and H. Oikawa: Scr. Mater. 22 (1988) 87-92.

31) R. Horiuchi and M. Otsuka: Mater. Trans. JIM 13 (1972) 284-293.

32) H. Takagi, M. Dao, M. Fujiwara and M. Otsuka: Mater. Trans. 47 (2006) 2006-2014 